\title{
NUEVOS REGISTROS DE AVES EN EL VALLE DEL RÍO MANTARO, HUANCAVELICA - PERÚ
}

\section{NEW BIRD RECORDS IN THE MANTARO RIVER VALLEY, HUANCAVELICA - PERU}

\author{
Miriam Torres $^{1}$, Margot Cuyos-Palacios ${ }^{2}$ y Flor Hernández ${ }^{3}$
}

\begin{abstract}
Resumen
Se realizó el Monitoreo Biológico en la Central Hidroeléctrica Cerro del Águila como parte del compromiso asumido en el Estudio de Impacto Ambiental (EIA), aprobado mediante Resolución Directoral $\mathrm{N}^{\circ}$ 049-2013-MEM/AAE en agosto de 2013. El área de estudio se ubica en los distritos de Colcabamba y Surcubamba, provincia de Tayacaja, departamento de Huancavelica, correspondiente al Valle de Mantaro. Las evaluaciones en campo se desarrollaron entre los años 2013 al 2016, durante las temporadas húmeda y seca de cada año; registrándose la ampliación de rango de distribución del Buco de Oreja Blanca (Nystalus chacuru), el Tirano-Todi de Vientre Perlado (Hemitriccus margaritaceiventer) y el Pinzón de Cresta Roja (Coryphospingus cucullatus). Especies cuyos registros en el área muestran variaciones tanto a nivel de frecuencia como abundancia entre temporadas y unidades de vegetación, esto posiblemente relacionado con la fase de construcción del proyecto.
\end{abstract}

Palabras clave: ampliación, distribución, rango, aves, Huancavelica.

\begin{abstract}
The biological monitoring of Cerro del Águila Hydroelectric Plant was developed as part of the commitments assumed in the Environmental Impact Assessment (EIA), which was approved through the Directorial Resolution No. 049-2013-MEM/AAE in August of 2013. The study area is located in Colcabamba and Surcubamba districts, Tayacaja province, Huancavelica department, corresponding to the Mantaro River Valley. Fieldwork was carried out between 2013 and 2016, during the wet and dry seasons of each year. As a result of this study, three species report extensions in their ranges of distribution: the White-Ear Buco (Nystalus chacuru), the Pearl Belly-Tyrant-Todi (Hemitriccus margaritaceiventer) and the Red-crested Finch (Coryphospingus cucullatus). These are species whose records in this area show variations in frequency and abundance between seasons and type of vegetation, possible related to the construction phase of the project.
\end{abstract}

Key words: extension, distribution, range, birds, Huancavelica.

\section{Introducción}

Como parte de los compromisos ambientales asumidos en el Estudio de Impacto Ambiental (EIA) del Proyecto Modificación de los componentes de la Central Hidroeléctrica Cerro del Águila, aprobado mediante Resolución Directoral $\mathrm{N}^{\mathrm{o}}$ 0492013MEM/AAE, Kallpa y Walsh Perú han desarrollado de manera conjunta los monitoreos biológicos entre los años 2013 al 2016, correspondientes a la fase de construcción de la Central Hidroeléctrica.

Como resultado de los monitoreos realizados, se registraron 3 especies de aves que reportaron ampliación en el rango de su distribución conocido hasta la fecha, siendo estas: el Buco de Oreja Blanca (Nystalus chacuru), el cual presenta una amplia distribución en Sudamérica (BirdLife, 2016a; Avibase, 2016a). Para Perú, ésta muestra una distribución fragmentada, siendo registrada en bosques secos y bordes de matorrales de los valles de Apurímac, Urubamba, del Mayo y en las sabanas de Pampa de Heath, entre los 1000 y $2000 \mathrm{~m}$ (Schulenberg et al., 2010). El Tirano-Todi de Vientre Perlado (Hemitriccus margaritaceiventer), el cual se distribuye desde el sureste de Venezuela hasta el norte de Argentina (BirdLife, 2016b; Avibase, 2016b), presentando para el caso de Perú poblaciones aisladas que suelen presentarse en áreas de matorrales de bosque secundario y bordes arbustivos de bosque en los valles del río Mayo, Huallaga, Chanchamayo y Urubamba, entre los 600 y $1600 \mathrm{~m}$ (Schulenberg et al., 2010). Y, por último, el Pinzón de Cresta Roja (Coryphospingus cucullatus), presente en varios países de Sudamérica (BirdLife, 2016c; Avibase, 2016c), mientras que para Perú, éste se presenta de manera irregular, registrándose en hábitats de matorrales secos de los 
valles del Marañón y del Urubamba, entre los 400 a 700 m (Schulenberg et al., 2010).

Ante lo expuesto, el presente trabajo tiene como objetivo dar a conocer la ampliación de los rangos de distribución de las especies previamente mencionadas, presentándose a su vez alcances de las variaciones poblacionales registradas para estas especies entre agosto del 2013 y febrero del 2016.

Área de estudio

El área del proyecto se localiza en el departamento de Huancavelica, provincia de Tayacaja, distritos de Colcabamba y Surcubamba. Como parte de la caracterización de la flora presente en el área se identificaron 5 unidades de vegetación:

- Bosque ralo caducifolio: el cual se ubica entre los 1990 y 2010 msnm. Reúne bosques fragmentados con presencia de cobertura vegetal dispersa. Entre las especies arbustivas características se encuentran Dodonea viscosa "chamana", Lantana balansae "titirica" y Lantana achyranthifolia "chanlla", mientras que el estrato arbóreo está representado por Eriotheca vargasii "pati", Cnidoscolus basiacanthus "inca itaña" y cactáceas columnares que logran alcanzar hasta los 12 metros de alto como Browningia hertlingiana "gigante" y Trichocereus peruvianus "sancay".

- Bosque denso perennifolio montano: ubicado entre los 1500 y $2010 \mathrm{msnm}$. Se emplaza en terrenos de pendiente moderada a fuerte, en donde éstos se presentan de forma fragmentada, especialmente en las partes bajas de las laderas, las que son empleadas como zonas de pastoreo de ganado vacuno. Entre las principales especies registradas para el estrato arbóreo destacan Leucaena trichodes y Anadenanthera colubrina, usadas como combustible (leña). El estrato arbustivo está conformado principalmente por Ipomoea vargasiana, Croton ruizianus, y especies de las familias Asteraceae y Verbenaceae; el estrado herbáceo está conformado por especies de las familias: Lamiaceae, Scrophulariaceae, Malvaceae, Amaranthaceae y Poaceae. La presencia de Cactaceas es muy frecuente, siendo los géneros más comunes Browningia, Cleistocactus y Opuntia.

- Matorral denso perennifolio montano: se presenta entre los 2100 y 3300 msnm. Se ubica en zonas de pendiente moderada con presencia de campos de cultivo y áreas de pastoreo, por lo cual es sometido con cierta frecuencia a procesos de "rosa y quema". Entre las especies vegetales registradas destacan Escallonia myrtilloides, Eucalyptus globulus, Brachyotum huancavelicae, Baccharis buxifolia, Baccharis latifolia, Rubus robustus, Dodonea viscosa "chamana" y Opuntia ficus indica "tuna".

- Matorral ralo caducifolio montano: se encuentra ubicado entre los 2500 y 2800 msnm. Reúne parches de matorral dispersos entre campos de cultivos empleados como "cercos vivos", en donde se registraron herbáceas como Bidens pilosa, Tagetes filifolia, Paspalum bonplandianum, entre otros.

- Vegetación ribereña: ubicada entre los 1300 y 3 $250 \mathrm{msnm}$. Agrupa a la vegetación presente en el cauce y bordes del río Mantaro.

\section{Metodología}

El monitoreo de la avifauna se centró en 8 estaciones de monitoreo, las que se encontraron distribuidas de la siguiente manera: 2 en el Bosque ralo caducifolio (Brc), 2 en el Matorral denso perennifolio montano (Mdp_m), 2 en la Vegetación ribereña $(\mathrm{Vr}), 1$ en el Matorral ralo caducifolio montano (Mrc_m) y 1 en el Bosque denso perennifolio montano (Bdp_m), (Figura 1, Tabla 1); todas estas evaluadas en temporada húmeda (enero-febrero 2014, enero 2015 y enerofebrero 2016) y temporada seca (agosto y octubre 2013, junio-julio 2014 y julio 2015).

Tabla 1: Ubicación de estaciones de monitoreo y unidades de vegetación evaluadas.

\begin{tabular}{|c|c|c|c|c|c|c|}
\hline Unidades de Vegetación & $\begin{array}{l}\text { Código } \\
\text { Unidad de } \\
\text { vegetación }\end{array}$ & $\begin{array}{l}\text { Estación de } \\
\text { Monitoreo }\end{array}$ & Este & Norte & $\begin{array}{l}\text { Elevación } \\
\text { (msnm) }\end{array}$ & $\begin{array}{l}\text { Componente } \\
\text { del Proyecto }\end{array}$ \\
\hline \multirow[t]{2}{*}{ Vegetación Ribereña } & $\mathrm{Vr}$ & EM-02 & 532711 & 8648035 & 1314 & Campamento \\
\hline & & & 8647975 & 532827 & 1314 & Platanal, \\
\hline \multirow{2}{*}{ Bosque Denso Perennifolio montano } & Bdp_mo & EM-01 & 533435 & 8647655 & 1471 & Limonal \\
\hline & & & 533644 & 8647800 & 1503 & \\
\hline Matorral ralo caducifolio montano & Mrc_m & EM-03 & 533573 & 8645612 & 2908 & Camino \\
\hline \multirow[t]{2}{*}{ Matorral denso perennifolio montano } & Mdp_m & EM-04 & 535515 & 8644511 & 3281 & proyectado \\
\hline & & & 535376 & 8644577 & 3371 & \\
\hline \multirow[t]{2}{*}{ Bosque ralo caducifolio } & $\mathrm{Brc}$ & EM-05 & 533888 & 8641482 & 1626 & Presa \\
\hline & & & 533410 & 8641028 & 1747 & \\
\hline \multirow[t]{2}{*}{ Matorral denso perennifolio montano } & Mdp_m & EM-06 & 537777 & 8640696 & 2115 & Fundición \\
\hline & & & 536752 & 8641172 & 2218 & \\
\hline \multirow[t]{2}{*}{ Bosque ralo caducifolio } & $\mathrm{Brc}$ & EM-08 & 538224 & 8636744 & 2167 & Embalse \\
\hline & & & 538329 & 9636309 & 2100 & \\
\hline \multirow{2}{*}{ Bosque ralo caducifolio } & $\mathrm{Brc}$ & EM-07 & 537288 & 8637480 & 2432 & \\
\hline & & & 536979 & 8637731 & 2142 & \\
\hline
\end{tabular}

Fuente: Walsh Perú S.A. 2013. 
Se empleó el método de conteo de puntos no limitado a la distancia (Bibby et al., 1985). En cada estación de muestreo se evaluaron 20 puntos de conteo, distanciados cada $100 \mathrm{~m}$, los cuales fueron ejecutados en las primeras horas de la mañana. Adicional a ello, se instalaron 10 redes de neblina $(12 \mathrm{~m}, 2.8 \mathrm{~m}$ y $36 \mathrm{~mm}$ de malla) por estación de muestreo, las que permanecieron activas por 3 días consecutivos. Las aves capturadas fueron fotografiadas y posteriormente liberadas, solo colectándose aquellas especies cuya identificación en campo no pudo ser determinada, contándose para ello con los permisos correspondientes (MINAGRI, 2013; SERFOR, 2015).

\section{Resultados}

El Buco de Oreja Blanca (Nystalus chacuru) fue registrado en todos los monitoreos realizados (2013 al 2016), indicando que es una especie constante en la zona (Figuras 2 y 3). Este fue avistado en 4 de las 5 unidades de vegetación identificadas, no registrándosele en el Matorral ralo caducifolio montano; sin embargo, su avistamiento en la Vegetación ribereña, solo se dio en el último monitoreo, lo que podría interpretarse como un registro fortuito, dado que solo se avistaron 2 individuos en esta zona. En cuanto a su abundancia total, esta especie fue predominante en el Bosque denso perennifolio montano (107 individuos) y Bosque ralo caducifolio (104 individuos); unidades de vegetación que reportaron las mayores variaciones poblacionales a lo largo de los monitoreos. En el caso del Matorral denso perennifolio montano, se observó un continuo descenso de individuos en la fase durante la construcción (2014, 2015), incrementándose los registros para finales de la etapa constructiva (2016). A nivel de temporalidad, los mayores valores de abundancia se reportan durante el inicio de la construcción (temporada seca 2013), los que posteriormente presentan variaciones entre temporadas y tipo de unidad de vegetación, pudiendo estos encontrarse relacionados con la fase (inicio, durante, final) de la etapa de construcción de la Central

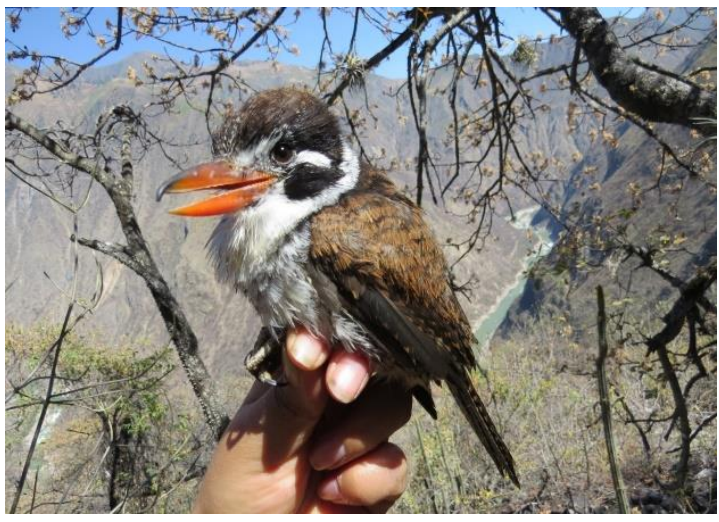

Figura 2. Individuo de Nystalus chacuru fotografiado en el Bosque Denso Perennifolio montano. Foto: Sonia Salazar.

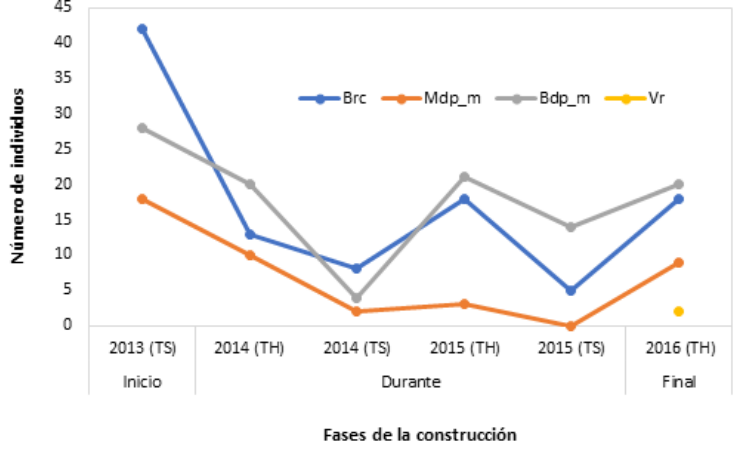

$\mathrm{MB}=$ Monitoreo biológico; $\mathrm{TH}=$ Temporada húmeda; $\mathrm{TS}=$ Temporada seca.

Unidades de vegetación: $\mathrm{Brc}=$ Bosque ralo caducifolio, Mdp_m= Matorral denso perennifolio montano, Bdp_m= Bosque denso perennifolio montano, $\mathrm{Vr}=$ Vegetación ribereña. Fuente: Walsh Perú S.A. 2016.

Figura 3. Variación de la abundancia de individuos registrados de Nystalus chacuru por unidad de vegetación y monitoreo.

Hidroeléctrica, así como cambios en composición vegetal dado por el cambio del uso del terreno.

Con relación al Tirano-Todi de Vientre Perlado (Hemitriccus margaritaceiventer), (Figuras 4 y 5), este fue registrado en todos los monitoreos realizados, así como en todas las unidades de vegetación muestreadas; sin embargo, su registro fue variable. En el Bosque ralo caducifolio se obtuvieron los mayores valores de abundancia total (118 individuos) y por temporada (49 individuos temporada húmeda 2016), aunque se encontró ausente para la temporada seca 2014. Similar escenario se da en el Bosque denso perennifolio montano, segunda unidad de vegetación más diversa (89 individuos en total), en la que se reporta variaciones en cuanto a su abundancia en la fase durante la construcción (2014), para después registrarse un incrementado de ésta para fines de la construcción (2016). En el caso del Matorral denso perennifolio montano, esta fue la única unidad de vegetación en la que se reportó un registro continuo, aunque también los menores valores de abundancia observándose, al igual que en el caso de las dos unidades de vegetación

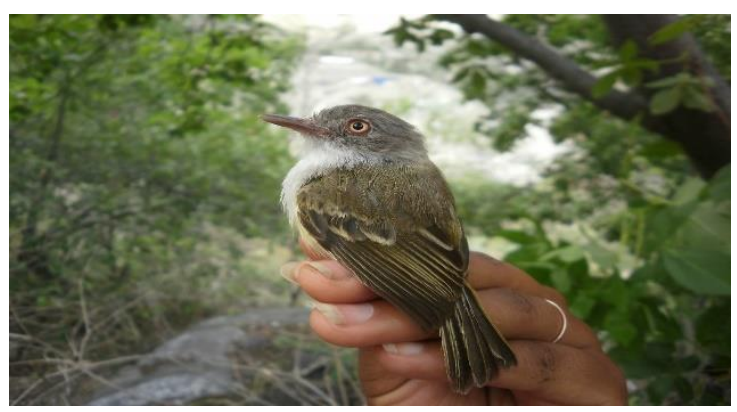

Figura 4. Individuo de Hemitriccus margaritaceiventer fotografiado en el Bosque Ralo Caducifolio. Foto: Sheila Figueroa. 


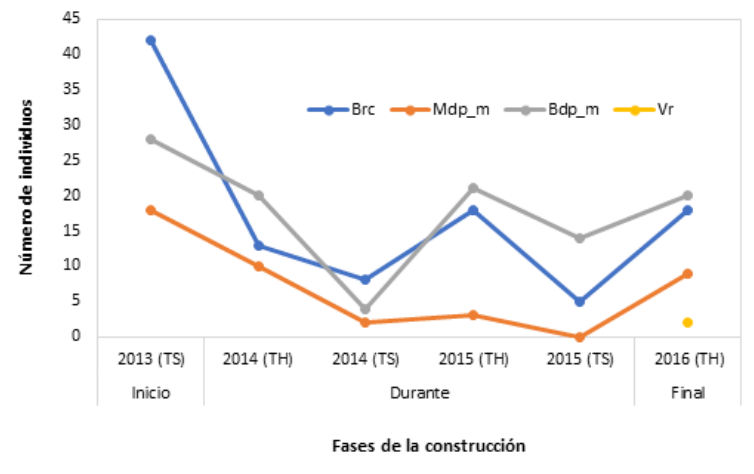

$\mathrm{MB}=$ Monitoreo biológico; $\mathrm{TH}=$ Temporada húmeda; $\mathrm{TS}=$ Temporada seca

Unidades de vegetación: $\mathrm{Brc}=$ Bosque ralo caducifolio, Mdp_m= Matorral denso perennifolio montano, Bdp_m= Bosque denso perennifolio montano, $\mathrm{Vr}=$ Vegetación ribereña. Fuente: Walsh Perú S.A. 2016.

Figura 5. Variación de la abundancia de individuos registrados de Hemitriccus margaritaceiventer por unidad de vegetación y monitoreo.

precedentes, un incremento de los registros para finales de la construcción (2016). Finalmente, en el caso de la Vegetación ribereña, el registro en esta área fue fortuito (avistada en 3 de los 6 monitoreos realizados), caracterizándose por reportarse un muy bajo número de individuos (2 individuos para el 2013).

El Pinzón de Cresta Roja (Coryphospingus cucullatus) (Figuras 6 y 7) presentó un comportamiento variable entre monitoreos y unidades de vegetación, reportando una presencia mínima en el área del proyecto (17 individuos). A nivel de unidades de vegetación, esta especie solo fue reportada para el Bosque denso perennifolio montano y Bosque ralo caducifolio, siendo en este último caso avistada exclusivamente durante el monitoreo correspondiente a la temporada húmeda 2015. En el caso del Bosque denso perennifolio montano, el número de individuos registrados fluctuó entre 1 y 4 ejemplares. Resultados que indicarían que esta especie no es común ni

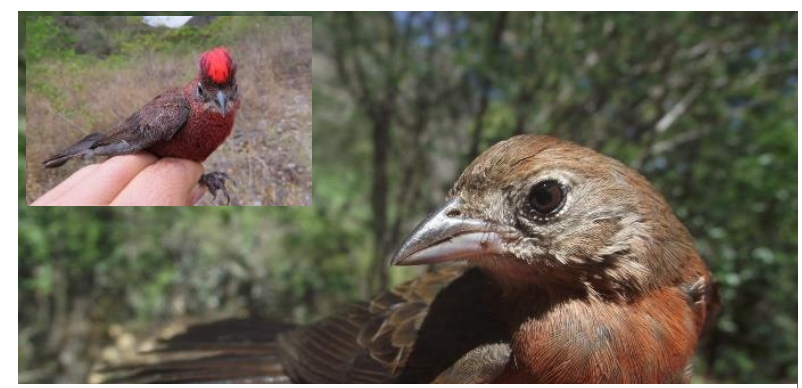

Figura 6. Individuo de Coryphospingus cucullatus fotografiado en el Bosque Denso Perennifolio Montano (Bdp_m). Fotos: Flor Hernández y Sonia Salazar.

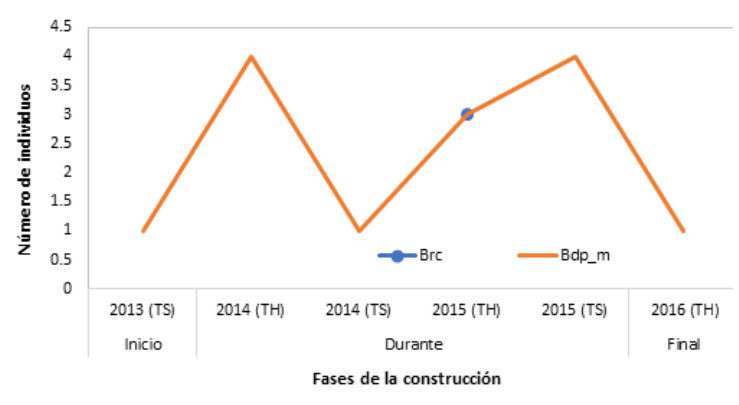

$\mathrm{MB}=$ Monitoreo biológico; $\mathrm{TH}=$ Temporada húmeda; $\mathrm{TS}=$ Temporada seca.

Unidades de vegetación: Brc= Bosque ralo caducifolio, Bdp_m= Bosque denso perennifolio montano.

Fuente: Walsh Perú S.A. 2016.

Figura 7. Variación de la abundancia de individuos registrados de Coryphospingus cucullatus por unidad de vegetación y monitoreo.

abundante en la zona del proyecto; sin embargo, cuando esta se registra, parecería preferir hábitats boscosos.

\section{Discusión}

Los registros presentados en este trabajo de Buco de Oreja Blanca (Nystalus chacuru), confirman la presencia de esta especie para el departamento de Huancavelica. Asimismo, esta especie ha sido reportada para el norte del Perú (valle del río Mayo), (Ayapi \& Ruiz, 2014); sur de Perú, específicamente en el Valle de Apurímac, Urubamba (Robbins et al., 2011) y Pampa de Heath (Schulenberg et al., 2010), destacándose en todos los casos el estatus de especie rara a poco común (Figura 8 ).

Robbins et al. (2011) reportaron un solo registro para esta especie, no pudiendo recabar evidencia concreta de su presencia en los bosques de piedemonte ubicados en la parte alta del valle del Urubamba. Ayapi \& Ruiz (2014) catalogaron a esta especie como rara en los alrededores de la ciudad de Moyobamba (cuencas de Mishiquiyacu-Rumiyacu y Almendra). Sin embargo, varios autores (Gurgel \& Quintas, 2013; Ayapi \& Ruiz, 2014) mencionan que $N$. chacuru está ampliamente distribuida en Sudamérica, pudiéndosele avistar en diferentes tipos de hábitats, los que pueden ir desde bosques hasta espacios abiertos. Este amplio espectro estaría relacionado a su condición de especie oportunista, además de presentar una dieta variada no restringiéndose solo a insectos (Crozariol \& Gomes, 2010; Gurgel \& Quintas, 2013), pudiendo incluso alimentarse de polluelos, siendo considerada como predador de nidos de otras especies (França et al., 2009).

Por otro lado, Daniel F. Lane colectó un ejemplar en el Valle Seco del Mantaro, Distrito Surcubamba, Provincia Tayacaja, Dpto. Huancavelica (12.09081 S, $74.69888 \mathrm{~W}$ ) en el 2010, reportando también la presencia de otros avistamientos en la carretera de Huancayo a Hucahocolpa (comunicación personal). 
Existen registros adicionales en el centro de Perú (eBird, 2016), como los reportados para el río Pampas, departamento Apurímac (Valqui \& Angulo, 2011).

De los registros obtenidos para el área del proyecto Cerro del Águila, se puede concluir que esta especie es común y abundante en la zona, presentando mayor afinidad a zonas boscosas, en concordancia con lo indicado por Schulenberg et al. (2010). Si bien esta especie reportó registros constantes a lo largo de los diferentes monitoreos realizados, no se ha podido establecer una tendencia en cuanto a su dinámica poblacional. Esto puede estar relacionado a los cambios que se han suscitado en el entorno producto de la construcción de la central hidroeléctrica. La mayor cantidad de registros coinciden con el inicio de la etapa de construcción, los cuales descienden a medida que se avanza con las obras del proyecto, para mostrar posteriormente un incremento en estos a medida que se llega al final de la construcción del proyecto, lo que podría indicar una recuperación de sus hábitats y consiguiente retorno de esta especie al área.

Los registros del Tirano-Todi de Vientre Perlado (Hemitriccus margaritaceiventer) reportados en el presente trabajo son los primeros documentados para el departamento de Huancavelica. Schulenberg et al. (2010) señalan que esta especie presenta registros aislados (parches) en el norte (valle del río Mayo y Huallaga), centro (valle del Chanchamayo) y sur (valle del Urubamba). En tanto que Daniel F. Lane la registró en el distrito de Pariahuanca, departamento de Junín (comunicación personal), (Figura 9).

$H$. margaritaceiventer es considerada bastante común, pero con distribución local para el Perú (Schulenberg et al., 2010). Patrón de distribución similar reportan Neris \& Colman (1991) en el monte espinoso del Chaco Central Paraguayo, Álvarez et al. (2009) en el Rincón de Franquía (Uruguay) y Stiles \& Tellería (1995) en la Sierra de Chiribiquete (Colombia).

Con relación a las unidades de vegetación, $H$. margaritaceiventer mostró un registro continuo en el Matorral denso perennifolio montano, mientras que fue discontinuo en las demás unidades de vegetación. Resultados que indicarían que posiblemente el Matorral denso perennifolio montano sea su unidad de vegetación preferencial. En cuanto a la frecuencia de su registro, esta especie sería aparentemente relativamente común a poco común en la zona. En tanto que, en el caso de su comportamiento con relación al proyecto, no se observó un patrón claro, pudiéndose indicar que mostró una relación directa positiva con la culminación de la etapa de construcción del proyecto.

De otro lado, los registros reportados para el Pinzón de Cresta Roja (Coryphospingus cucullatus), corresponden a los primeros para el departamento de Huancavelica. Especie considerada bastante común, pero distribuida en parches en el valle del Marañón y Urubamba, según Schulenberg et al. (2010), (Figura $10)$.
Son varios los autores que reportan la ampliación de distribución de esta especie en Sudamérica y su abundancia local en las áreas que evaluaron (Zotta, 1941; Tierno de Figueroa \& Padial, 2005; Girando et al., 2006; Della-Flora et al., 2010), cuyo hábitat son los bosques secos, sabanas, selvas, matorrales, áreas húmedas, plantaciones y zonas rurales (De la Peña, 2011).

Para el área del proyecto, esta especie reportó una presencia mínima, pudiéndosele considerársele rara para la zona. Asimismo, al solo ser registrada de forma continua en un tipo de unidad de vegetación (Bosque denso perennifolio montano), este resultado indicaría que presenta requerimientos de hábitat específicos, los que podrían encontrarse relacionado a zonas boscosas con coberturas vegetales densas, como la reportada para el área de estudio. Por otro lado, si bien esta especie también fue avistada en el Bosque ralo caducifolio, su registro fue puntual (temporada húmeda 2015), por lo que no sería representativo para esta zona. En tanto que, a nivel del patrón de distribución y abundancia presentada por esta, no se observó ningún patrón claro, por lo que no se pudo encontrar relación entre su distribución y fase del proyecto.

Ante lo expuesto, los resultados reportados permiten confirmar la ampliación del rango de distribución de las 3 especies mencionadas. De acuerdo a la frecuencia de su registro y unidad de vegetación en la que se avistaron, su presencia en la zona es constante, aunque variable en cuanto a su abundancia. De otro lado, las fases constructivas del proyecto, aunadas a las variaciones en el hábitat por el cambio de uso del terreno (ampliación de las áreas de cultivo y actividades constructivas), habrían influido en la dinámica poblacional de estas especies. Es así que, para el caso de las especies Buco de Oreja Blanca (Nystalus chacuru) y Tirano-Todi de Vientre Perlado (Hemitriccus margaritaceiventer), estas reportaron un incremento en sus poblaciones al final de la fase constructiva; en tanto que en el caso del Pinzón de Cresta Roja (Coryphospingus cucullatus), este muestra un comportamiento inverso, pero al reporta una mínima presencia en el área, estos resultados deben ser considerados con precaución. Se hace necesario para todos los casos la continuidad de las actividades de monitoreo biológico en el área del proyecto, a fin de realizar seguimiento a las variaciones estacionales y temporales de estas especies.

\section{Agradecimientos}

Expresamos nuestro agradecimiento a la empresa Cerro del Águila S. A., propiedad de Kallpa Generación S.A por permitirnos revisar y publicar esta información. También agradecer a nuestros colegas Nadia Sánchez por su apoyo en la gestión del Proyecto; William Lopez e Isabel Altez por su apoyo en la parte cartográfica; Daniel F. Lane por la revisión y aportes al presente documento; Gilda Bianco por su revisión en la 
redacción del resumen en el idioma de inglés y; a Sheila Figueroa, Sonia Salazar, Aiko Valencia y Vania Tejeda por su participación en campo.

\section{Literatura citada}

Álvarez S., Menéndez J. \& Rocca P. 2009. Las Aves de Rincón de Franquía. Informe técnico. GRUPAMA (Grupo para la Protección Activa del Medio Ambiente).

Avibase. 2016a. Avibase: The world bird database [web application]. Species factsheet: Nystalus chacuru. Consultado en: https://avibase.bsceoc.org/species.jsp?lang=ES\&avibaseid=74AF2CB6\&se $\mathrm{c}=$ summary \&ssver=1. Fecha de consulta: 11/03/2016.

Avibase. 2016b. Avibase: The world bird database [web application]. Species factsheet: Hemitriccus margaritaceiventer. Consultado en: https://avibase.bsceoc.org/species.jsp?lang=ES\&avibaseid=6B4E9E6E\&se $\mathrm{c}=$ summary\&ssver $=1$. Fecha de consulta: 11/03/2016.

Avibase. 2016c. Avibase: The world bird database [web application]. Species factsheet: Coryphospingus cucullatus. Consultado en: https://avibase.bsceoc.org/species.jsp?avibaseid=632FA6BD. Fecha de consulta: 11/03/2016.

Ayapi J.A. \& Ruiz S.A. 2014. Avifauna de las microcuencas de Almendra, Mishquiyacu y Rumiyacu. Informe técnico del Proyecto BIOCUENCAS.

Bibby C.J., Phillips B.N. \& Seddon A.J.E. 1985. Birds of restocked conifer plantations in Wales. Journal of Applied Ecology, 22: 619-633.

BirdLife International. 2016a. Species factsheet: Nystalus chacuru. Acceso en: http://datazone.birdlife.org/species/factsheet/226822 64. Fecha de consulta: 11/03/2016.

BirdLife International. 2016b. Species factsheet: Hemitriccus margaritaceiventer. Acceso en: http://datazone.birdlife.org/species/factsheet/Pearlyvented-Tody-Tyrant. Fecha de consulta: 11/03/2016.

BirdLife International. 2016c. Species factsheet: Coryphospingus cucullatus. Acceso en: http://datazone.birdlife.org/species/factsheet/red-crestedfinch-coryphospingus-cucullatus. Fecha de consulta: 11/03/2016.

Crozariol M.A. \& Gomes F.B. 2010. Insetívoro ou Oportunista? A dieta do joão-bobo, Nystalus chacuru (Galbuliformes: Bucconidae). In: eBird. eBird: An online database of bird distribution and abundance [web application]. Fecha de consulta: 11/03/2016.

De la Peña M. 2011. Una de 1000 aves argentinas, Brasita de Fuego (Coryphospingus cucullatus). Revista electrónica de divulgación del Museo Provincial de Ciencias Naturales "Florentino Ameghino". Ministerio de Innovación y Cultura.
Della-Flora F., Catarina M. S., Duarte G., Turchielo C. \& Canto-Dorow T. 2010. Revista Brasileira de Ornitología, 18 (4): 344-346.

eBird. 2016. The Cornell Lab. of ornithology. Mapa de distribución de Nystalus chacuru. Acceso en: https://ebird.org/map/whepuf1?neg=true\&env.minX=\&e nv.min $Y=\&$ env. $\max X=\&$ env. $m a x Y=\& z h=$ false $\& g p=$ fal $\underline{\text { se\&ev }=\mathrm{Z} \& \mathrm{mr}=1-}$

$12 \& b m o=1 \&$ emo $=12 \& y r=a l l \& b y r=1900 \&$ eyr $=2018$. Fecha de consulta: 05/03/2016.

França L.F., Sousa N.O., Dos Santos L.R., Duca C.G.D.T., Borges F.J., Lopez L.E., Manica L.T., Palva L.V., De Medelros R.C. \& Marini M.A. 2009. Passeriformes: nest predators and prey in a Neotropical Savannah in Central Brazil. Zoología, 26(4): 799-802.

Giraudo L., Kufner M., Torres R., Tamburini D., Briguera V. \& Cavier G. 2006. Avifauna del bosque chaqueño oriental de la provincia de Córdoba, Argentina. Ecología Aplicada, 5(1,2): 127-136. DOI: http://dx.doi.org/10.21704/rea.v5i1-2.326.

Gurgel G. \& Quintas S. 2013. (Piciformes: Bucconidae) predando Ameivula ocellifera (Squamata: Sauria: Teiidae). Heringeriana, 7(2): 177-178.

MINAGRI. 2013. Resolución Directoral N N ${ }^{\circ}$ 0282-2013MINAGRI-DGFFS-DGEFFS: se autoriza a Cerro del Águila la colecta de especímenes de flora y fauna en el marco del proyecto Central Hidroeléctrica Cerro del Águila.

Neris N. \& Colman F. 1991. Observaciones de aves en los alrededores de Colonia Neuland, departamento Boquerón, Paraguay. Bol. Mus. Nac. His. Nat. Paraguay, 10: 1-10.

Robbins M.B., Geale D., Walker B., Davis T., Combe M., Eaton M.D. \& Kennedy K.P. 2011. Foothill avifauna of the upper Urubamba Valley, dpto. Cusco, Perú. Cotinga, 33: $34-45$.

SERFOR. 2015. Resolución de Dirección General N ${ }^{\circ} 0011$ 2015-SERFOR-DGGSPFFS: se autoriza a Cerro del Águila la colecta de especímenes de flora y fauna en el marco del proyecto Central Hidroeléctrica Cerro del Águila.

Schulenberg T., Stotz D., Lane D., O’ Neill J. \& Parker T. 2010. Aves del Perú. Serie Biodiversidad CORBIDI 01. Lima, Perú.

Stiles F.G. \& Tellería J.L. 1995. Observaciones sobre la composición, ecología, y zoogeografía de la avifauna de la Sierra de Chiribiquete, Caqueta, Colombia. Caldesia, 17 (82-85): 481-500.

Tierno de Figueroa J.M. \& Padial J.M. 2005. Avifauna invernal en ambientes de Chaco y Pantanal en Bahía Negra (norte de Paraguay). Hornero, 020(02): 153-162.

Valqui T. \& Angulo F. 2011. Registro de Nystalus chacuru en Rio Pampas, debajo de Mara Mara, Apurímac, PE. En eBird. The Cornell Lab. of ornithology. https://ebird.org/peru/view/checklist/S8766098.

Zotta A.R. 1941. Lista sistemática de las aves argentinas. Hornero, 8(1): 137-153. 


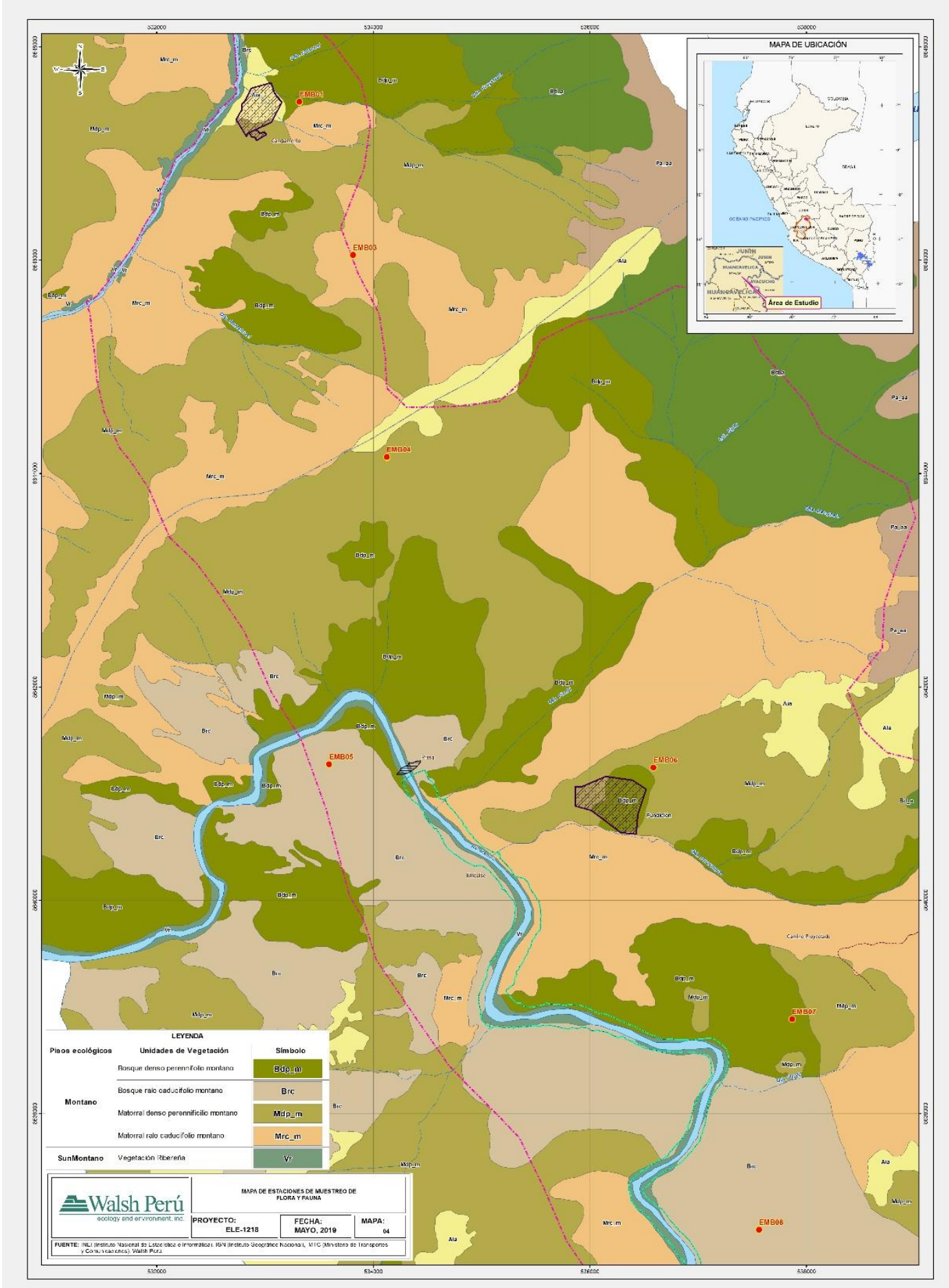

Figura 1. Mapa del área de estudio. 


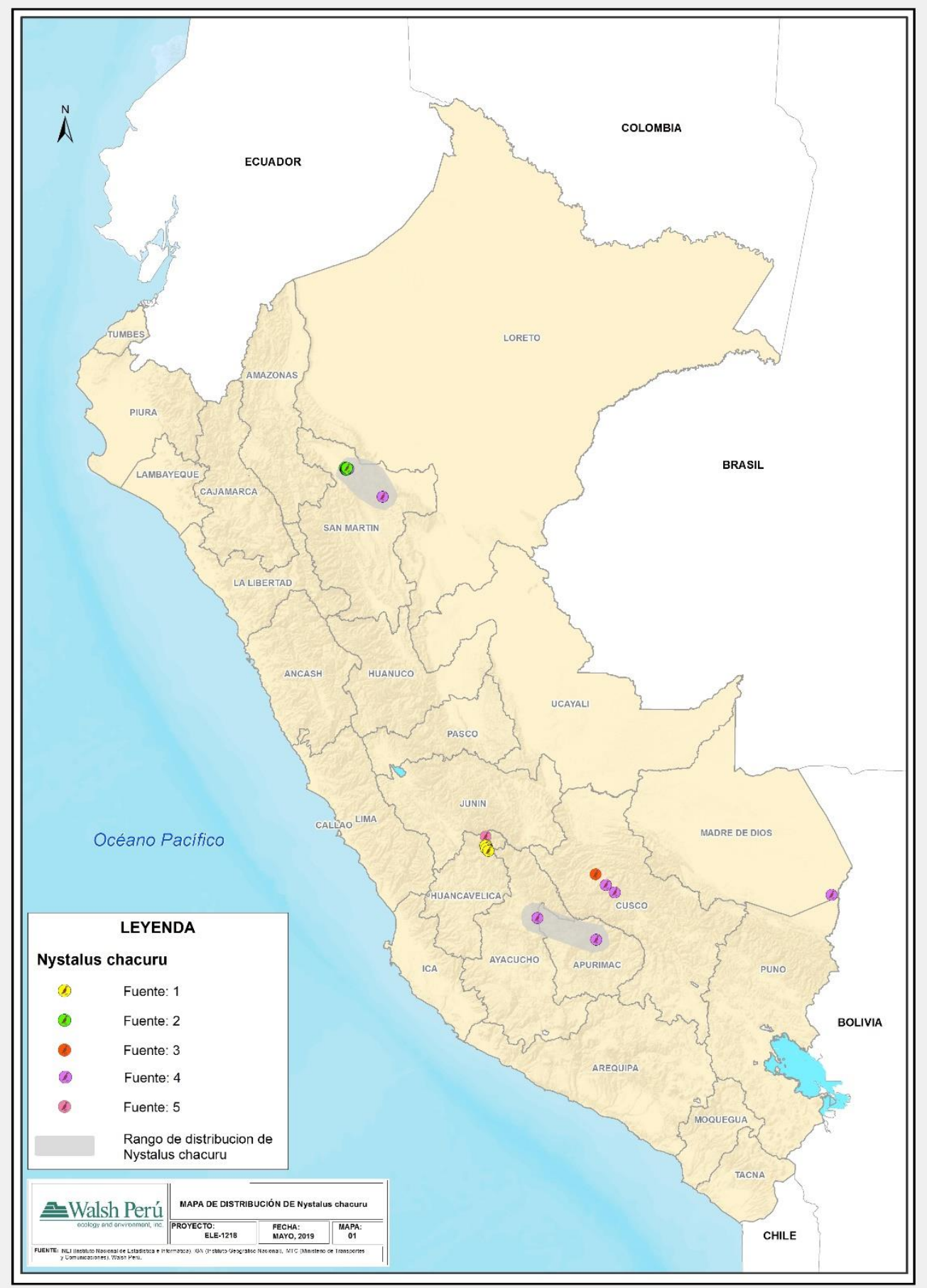

Fuente 1: Registros reportados en el presente trabajo; Fuente 2: Ayapi \& Ruiz (2014); Fuente 3: Robbins et al. (2011); Fuente 4: Schulenberg et al. (2010); Fuente 5: Registros de Daniel F. Lane.

Elaborado por: Walsh Perú S.A., 2019.

Figura 8. Mapa de distribución de Nystalus chacuru. 


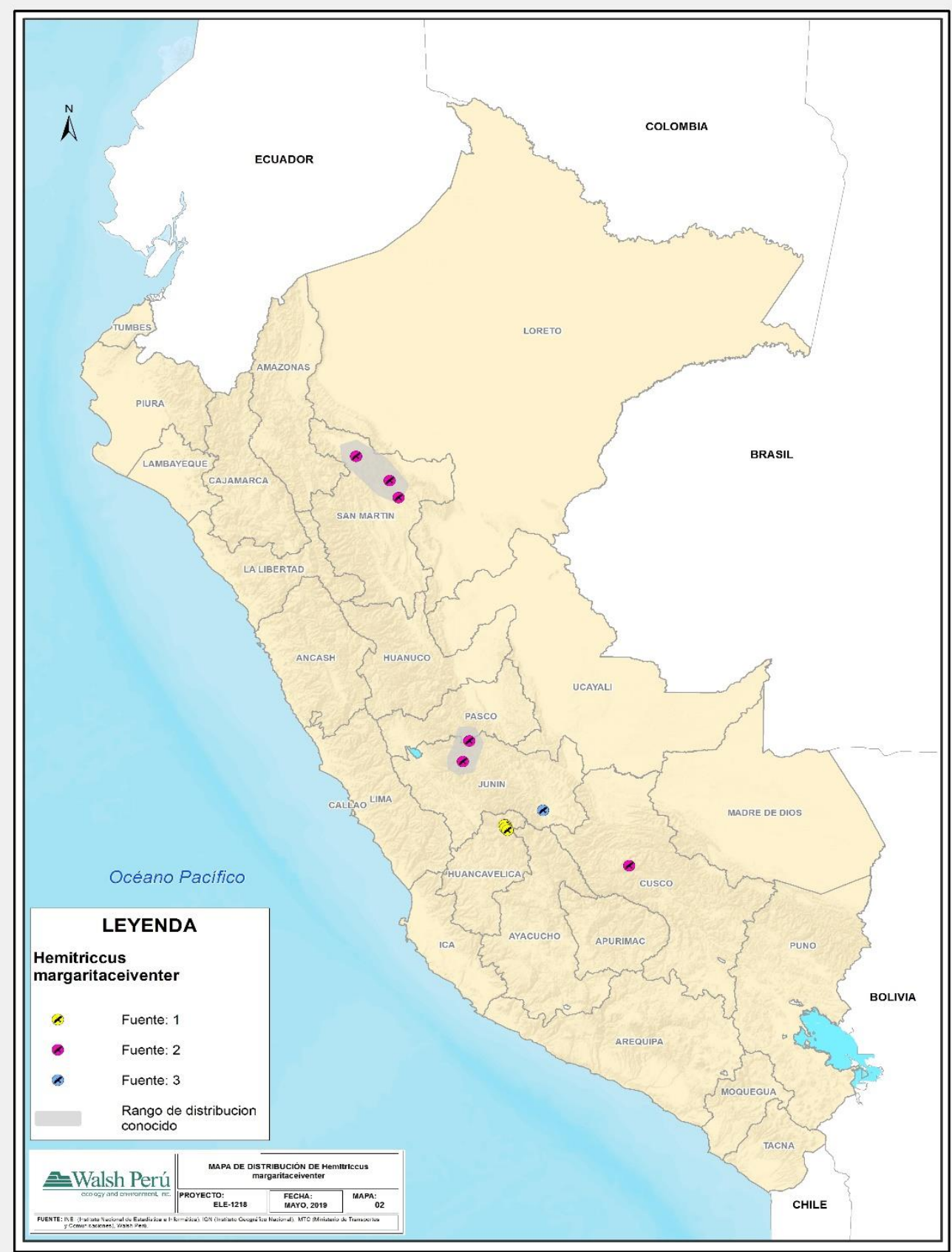

Fuente 1: Registros reportados en el presente trabajo; Fuente 2: Schulenberg et al. (2010); Fuente 3: Registros de Daniel F. Lane

Elaborado por: Walsh Perú S.A., 2019.

Figura 9. Mapa de distribución de Hemitriccus margaritaceiventer. 


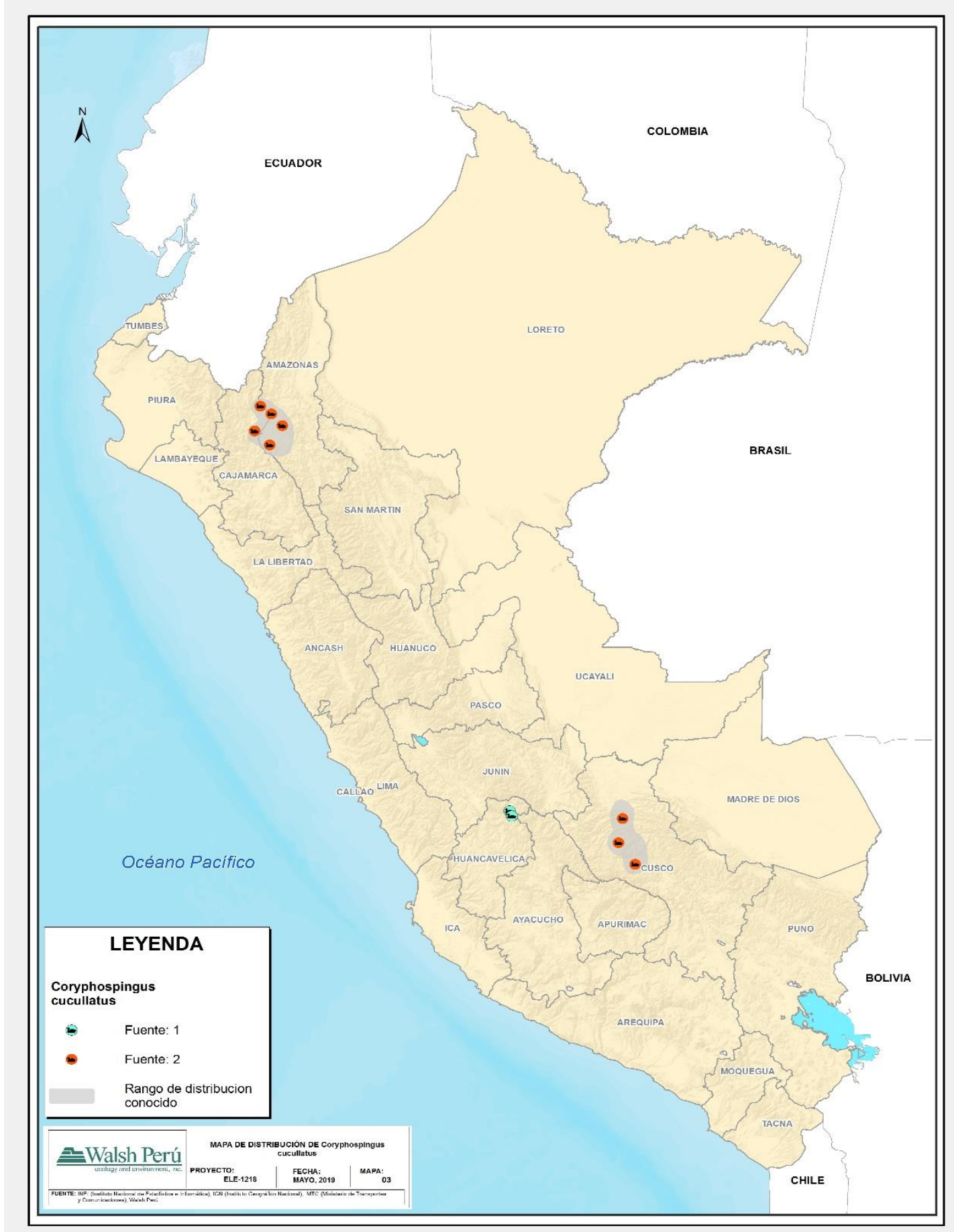

Fuente 1: Registros reportados en el presente trabajo; Fuente 2: Schulenberg et al. (2010). Elaborado por: Walsh Perú S.A., 2019.

Figura 10. Mapa de distribución de Coryphospingus cucullatus.

\footnotetext{
${ }^{1}$ Walsh Perú S.A. Autor para correspondencia: Miriam Torres: mtorres@ walshp.com.pe.

${ }^{2}$ Walsh Perú S.A

${ }^{3}$ Estación Biológica Río Santa Eulalia (STEU) - Centro de Ornitología y Biodiversidad (CORBIDI).
} 